\title{
La prévision de la défaillance des entreprises marocaines : Analyse empirique
}

\author{
Fatiha ABID and Saleh DRIYASSE \\ ${ }^{1}$ Doctorante en économie, Faculté des Sciences Juridiques Economiques et Sociales, Université Cadi Ayyad, \\ Marrakech, Maroc \\ ${ }^{2}$ Doctorant en économie, Ecole Nationale de Commerce et Gestion, Université Mohammed 5, Kénitra, Maroc.
}

\begin{abstract}
Résumé : La prévision de la défaillance des entreprises fait l'objet de nombreux travaux empiriques. Elle se fonde sur l'analyse économique et financière d'entreprises défaillantes et d'entreprises non défaillantes, afin de déterminer les variables, principalement comptables, qui distinguent au mieux les deux catégories de firmes. Nous proposons un modèle afin de rendre compte de l'efficacité relative des différentes méthodes de classification utilisées. Dans ce but, nous exposons la démarche commune tout en mettant en évidence les différentes modalités d'application empirique. Nous présentons le principe des techniques disponibles et une comparaison de leur performance, en mettant l'accent, de manière non exhaustive, à la fois sur les études fondatrices et sur les études les plus récentes.
\end{abstract}

Mots clés : Défaillance, Analyse discriminante, régression logistique.

\begin{abstract}
The prediction of business failure is the subject of much empirical work. It is based on the economic and financial analysis of failing and non-failing firms, in order to determine the variables, mainly accounting variables, that best distinguish the two categories of firms. We propose a model to account for the relative efficiency of the different classification methods used. To this end, we present the common approach while highlighting the different empirical applications. We present the principle of the available techniques and a comparison of their performance, focusing, in a non-exhaustive way, on both the founding studies and the most recent ones.
\end{abstract}

Keywords : Failure, Discriminant analysis, Logistic regression.

\section{Introduction :}

La défaillance est un fait brutal qui menace la continuité des entreprises. Elle constitue un événement de nature financière à caractère juridique incontournable, et dont les origines sont principalement économiques. Bien que la défaillance constitue un risque pour l'entreprise, c'est une véritable menace des économies. Le traitement des secteurs de l'industrie et du commerce du Maroc a 
montré que les PME sont les plus dominantes avec un pourcentage de 90\%, dont la mondialisation et l'environnement économique constituent leurs véritables enjeux. En effet toute entreprise est menacée par la défaillance, dont le pourcentage du risque se diffère d'une entreprise à une autre.

Du coté juridique, la loi marocaine de 1966 traite le sujet de la défaillance, cette loi prévoit également les difficultés, liquidation des entreprises, et redressements.

Des institutions juridiques ayant compétence en matière de procédures collectives ont été créées en 1997 pour faciliter l'application cohérente de la nouvelle législation, à savoir le redressement ou la liquidation qui sont régis par une procédure unique entraînant l'application de l'une ou de l'autre solution, ce qui a amélioré considérablement la résolution des conflits commerciaux et a participé à un traitement plus cohérent et efficace des litiges par le système judiciaire. Cependant l'analyse des difficultés des entreprises par les débiteurs qui bénéficient d'une durée parfois exagéré et peuvent faire des recours corrompu, menace l'efficacité du nouveau régime. Il parait évident que le défi du législateur est d'instaurer des mécanismes de résolution de la cessation des paiements adaptés à l'environnement entrepreneurial marocain. Cela permet d'une part de lutter contre la défaillance des entreprises, en instaurant des outils de traitement ex ante à la cessation des paiements. D'autre part les procédures de résolution des difficultés doivent répondre aux exigences des parties prenantes de la défaillance, à savoir les créanciers et les débiteurs.

L'accroissement actuel de la taille des entreprises en défaillance, donc des montants de dettes concernés, rappelle vivement la nécessité de prévoir la défaillance. L'évaluation quantitative du risque de défaillance, s'est principalement développée à partir de la fin des années soixante. L'approche la plus fréquente consiste à recourir à L'analyse financière afin de déterminer les variables, principalement comptables, qui différencient au mieux les entreprises défaillantes et les entreprises non défaillantes. L'objectif est d'établir une relation statistique stable entre les variables explicatives retenues et l'appartenance des entreprises à l'un des deux groupes. Différents outils sont à la disposition des auteurs, dont le plus fréquemment utilisé est L'analyse discriminante linéaire, mais les méthodes alternatives sont nombreuses.

Cet article s'inscrit dans le prolongement des travaux sur la défaillance des entreprises. Il vise à proposer un modèle adéquat d'aide à la décision. L'intérêt de cette recherche est d'identifier les outils de prévision de la défaillance des entreprises marocaines et de proposer un meilleur modèle d'anticipation de la détresse financière.

L'objectif principal de ce travail consiste à faire une comparaison de la capacité prédictive des trois méthodes utilisée à savoir : La régression logistique, l'arbre de décision et l'analyse discriminante. Pour cet objectif, une étude empirique a été réalisée dans l'intérêt de construire un modèle adapté au contexte entrepreneurial marocain, permettant de prévoir et mesurer le risque de la défaillance d'une entreprise en faisant appel à des variables explicatifs de la défaillance des entreprises. 
A ce titre, un échantillon d'entreprises marocaines sera être analysé sur la base de certaines variables quantitatives pour formuler un modèle de prévision de la régression logistique, l'arbre de décision et l'analyse discriminante. Pour tester la capacité prédictive de ces trois méthodes nous proposons à la fin de cet article un tableau récapitulatif des principales études faites, en privilégiant celles qui permettent une comparaison rigoureuse des différentes techniques utilisé. Et nous concluons en proposant une synthèse de l'efficacité de chacune des techniques présentées.

Le nombre des entreprises concernées par la défaillance, rappelle vivement la nécessité de prévoir le phénomène, ceci passe par l'anticipation des graves difficultés économiques et financières qu'une entreprise est susceptible de rencontrer. Cela implique notamment une estimation précise du risque de défaut, et donc une véritable maîtrise des méthodes d'évaluation et de prévision des risques.

Les techniques de prédiction de la faillite sont des moyens qui permettent de déterminer si une entreprise est défaillante dans un horizon plus ou moins lointain. Ces outils fonctionnent à partir des informations financières des entreprises. Les informations financières sont extraites de la comptabilité de ces entreprises. Le but de la méthode quantitative d'évaluation du risque de la défaillance est de dresser une relation statistique stable entre les variables explicatives retenues et l'appartenance des entreprises à l'un des deux groupes, à savoir défaillantes ou non-défaillantes.

Les techniques de prévision de défaut donnent des résultats acceptables, donc il est obligatoire de décrire les différentes modalités d'application empirique et la méthodologie, la construction de l'échantillon, la sélection des variables explicatives et l'approche de validation des résultats.

\section{Les modèles de prévision de la défaillance des entreprises}

Pompe et BILDERBEEK (2005) ${ }^{1}$, testent une hypothèse sur le pouvoir prédictif des différentes catégories de rapport pendant les périodes successives avant la faillite, ainsi que la relation entre l'âge d'une entreprise et la prévisibilité de la défaillance en utilisant les données pour les PME et ont conclu que chaque rapport enquêté avait un certain pouvoir prédictif, et il n'y a pas d'ordre fixe dans le pouvoir de prédiction des différentes catégories de ratios financiers. Et les rapports indiquent l'efficacité prédictives similaires 5 ans avant l'échec. Ils ont également constaté que la défaillance des jeunes entreprises est plus difficile à prévoir que la défaillance des entreprises bien établies, donc ils ont obtenu de meilleurs résultats en ayant un modèle distinct pour chaque catégorie d'âge. ${ }^{2}$

\footnotetext{
${ }^{1}$ Pompe \& BILDERBEEK (2005), cite in SAEIDEH ALIAKBARI, Prediction of Corporate Bankruptcy for the UK Firms in Manufacturing Industry, Department of Economics and Finance, School of Social Sciences MSc in Finance and Investment, Brunel University, September 2009.

${ }^{2}$ SAEIDEH ALIAKBARI, Prediction of Corporate Bankruptcy for the UK Firms in Manufacturing Industry, Department of Economics and Finance, School of Social Sciences MSc in Finance and Investment, Brunel University, September 2009.
} 


\subsection{La régression logistique}

Lorsque la variable à expliquer est qualitative on peut appliquer la régression logistique pour estimer ses coefficients, ce variable à expliquer admet un attribut et non pas une valeur numérique. On peut introduire une codification qui nous permet de représenter les différents attributs Lorsque la variable à expliquer est qualitative, En présente le code « 0 » si l'attribut est absent chez l'individu étudié et « 1 » s'il est présent. L'objectif du modèle n'est pas de prédire une valeur numérique de la variable à expliquer, ce qui n'aurait pas grand sens, mais de prévoir la probabilité que cet individu ait la caractéristique associée au code « $1 »$ de la variable à expliquer, connaissant les valeurs prises par les variables explicatives chez un individu donné. Dans le cas qui nous intéresse, il s'agit de connaître la probabilité de défaillance d'une entreprise, sachant ses caractéristiques comptables et financières ${ }^{3}$.

La justification de choix de la régression logistique à la place de la régression linéaire ordinaire, C'est que pour la régression linéaire, la variable qu'on veut expliquer est quantitative. De plus, la régression linéaire ordinaire n'est pas applicable si les erreurs ne sont pas distribuées normalement ou la variance des erreurs n'est pas constante. Pour contrer ces difficultés, une transformation « Logit » est nécessaire. Le modèle s'écrit alors de la manière suivante :

La probabilité d'occurrence selon la formule logistique s'écrit :

$$
E(x)=\pi(x)=P(Y=1 / X=x)=\frac{e^{\beta_{0}+\beta_{1} x_{1}+\ldots+\beta_{k} x_{k}}}{1+e^{\beta_{0}+\beta_{1} x_{1}+\ldots+\beta_{k} x_{k}}}
$$

$\mathrm{Y}$ est la variable à expliquer c'est la faillite et $\mathrm{X}$ ce sont les variables explicatives ce sont les ratios de chaque entreprise.

Equation équivalence par transformation à la fonction Logit :

$$
\ln \left(\frac{P(Y=1 / X)}{P(Y=0 / X)}\right)=\beta_{0}+\beta_{1} X_{1}+\beta_{2} X_{2}+\ldots+\beta_{k} X_{k}
$$

\section{- Les propriétés de la régression logistique :}

La fonction logistique est monotone croissante ou décroissante, selon le signe de 1 d'une part, et que la fonction logistique est presque linéaire lorsque $\mathrm{E}(\mathrm{Y})$ est entre 0,2 et 0,8 et s'approche graduellement de 0 et 1 aux deux extrémités du support de $X$. 
Aussi, la distribution logistique est symétrique $\mathrm{E}(-\mathrm{Y})=1-\mathrm{E}(\mathrm{Y})$, et de moyenne nulle (voir figure $)^{4}$ :

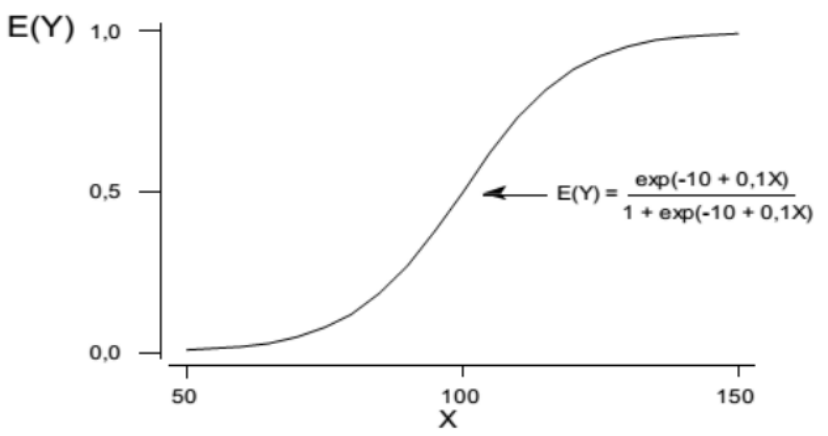

Figure 3: la fonction logistique

Une autre propriété de la régression logistique est qu'elle n'impose pas que les variables indépendantes suivent une loi normale.

Test de signification des coefficients

\subsubsection{Test de WALD}

Le test de signification des coefficients du modèle de régression logistique, se fait par le biais du test de WALD. Ce test est le plus utilisé dans la validation des coefficients du modèle de régression logistique, elle utilise une statistique $\mathrm{W}$ appelée statistique de WALD qui s'interprète comme un chi-deux, au seuil de signification de 5\%. Cette statistique est définie par :

Statistique utilisée :

$W A L D_{j}=\frac{\hat{\beta}_{j}^{2}}{\left(\text { erreur type de } \beta_{\mathrm{j}}\right)^{2}}$

\subsubsection{L'évaluation de la régression : les pseudo-R2}

Une question cruciale est de pouvoir déterminer si le modèle obtenu est "intéressant" ou non. Le premier à pouvoir trancher est l'expert. En se basant sur les contraintes du domaine, il peut nous dire si le modèle est suffisamment concluant.

${ }^{4}$ Ricco Rakotomalala : Pratique de la Régression Logistique (Régression Logistique Binaire et Polytomique). 


$$
\text { Pesudo }-R^{2}=1-\left[\frac{-2 L(\text { cte }, X)}{-2 L(\text { cte })}\right]
$$

Il existe d'autre moyens d'évaluation parmi lesquels notant :

$\mathrm{R}^{2}$ de Cox Snel

$$
R^{2}=1-\left[\frac{L(c t e)}{L(c t e, X)}\right]^{\frac{2}{n}} \operatorname{MaxR}^{2}=1-\left[\frac{-2 L(c t e, X)}{-2 L(c t e)}\right]
$$

$\mathrm{R}^{2}$ ajusté de Nagelkerke

$$
R_{a d j}^{2}=\frac{R^{2}}{R_{\max }^{2}}
$$

\subsubsection{Courbe de ROC}

Le graphe de ROC permet d'étudier les fluctuations de la sensibilité et de la spécificité pour différentes valeurs du seuil de discrimination. Le terme de courbe roc peut être envisagé comme une "courbe de caractéristiques d'efficacité".

$\checkmark$ La sensibilité : représente la probabilité de bien détecter un positif. Dans le cas de notre projet elle représente la proportion des vrais risqués.

$\checkmark$ La spécificité : représente la probabilité de bien détecter un négatif. Dans notre cas elle représente la proportion des vrais non risqués.

La courbe de roc est primordiale pour évaluer les performances d'un modèle de prédiction et pour savoir si le modèle est globalement significatif.

\section{- Construction de la courbe du ROC :}

On porte sur l'axe des abscisses, la variable «1-spécificité », cette variable est égale à l'effectif des faux positifs parmi les non défaillants. Sur l'axe des ordonnées, on retrouve la sensibilité, égale à l'effectif de vrais positifs parmi les défaillants. La courbe se construit de façon empirique avec le calcul de la sensibilité puis la spécificité pour différents niveaux de seuils de discrimination. 


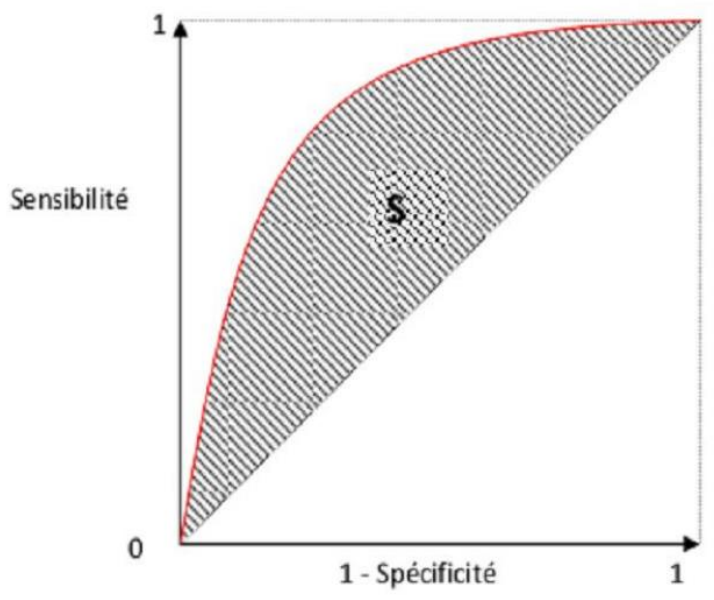

Figure 4 : Courbe de ROC.

-Aire sous la courbe ROC : L'aire sous la courbe de ROC est un estimateur de l'efficacité globale du test, en absence de discrimination, l'aire est de 0.5, cela veut encore dire que le modèle n'est pas meilleur qu'un modèle aléatoire. Par contre, si le test est parfaitement discriminatif, l'aire sera de 1 . Si on note par Z la zone sous la courbe de ROC on a :

$0,9<\mathrm{Z}<1,0:$ discrimination excellente

$0,8<\mathrm{Z}<0,9$ : bonne discrimination

$0,7<\mathrm{Z}<0,8:$ discrimination correcte

$0,6<\mathrm{Z}<0,7$ : discrimination médiocre

$0,5<\mathrm{Z}<0,6:$ mauvaise discrimination

\subsubsection{Indice de Kappa}

En statistiques, le test du Kappa mesure l'accord entre observateurs lors d'un codage qualitatif en catégories. Kappa (\%) : compare le classement obtenu à un classement obtenu par hasard. Le calcul du Kappa se fait de la façon suivante :

$$
\begin{aligned}
& \text { Kappa }=100 \times \frac{p(\text { correct })-p(\text { hasard })}{1-p(\text { hasard })} \text { avec } \\
& p(\text { correct })=\frac{(T P+T N)}{(T P+T N+F P+F N)} \\
& p(\text { hasard })=\frac{\left(T P-\frac{(T P+F P)}{2}\right)+\left(T N-\left(\frac{(T N+F N)}{2}\right.\right.}{(T P+T N+F P+F N)}
\end{aligned}
$$


Le classement est parfait si Kappa $=100 \%$ et pas meilleur que celui obtenu par hasard si Kappa $=0 \%$. Pour calculer l'indice de Kappa, nous présentons les informations suivantes :

TP : « True Positives » ou «Vrais positifs »

$\mathrm{TN}:$ « True Negatives » ou « Vrais négatifs »

FP : «False Positives » ou « Faux positifs»

FN : «False Negatives » ou «Faux négatifs »

Par exemple un « TP » est un «bon » client classé « bon », «TN » est un mauvais client classé « mauvais », un « FP » est un « mauvais » client classé « bon » et un «FN » est un bon » client classé « mauvais ». Avec ces notations nous pouvons élaborer le tableau suivant :

Tableau 6 : les notations élaborées pour le calcul de l'indice de Kappa.

\begin{tabular}{|c|c|c|c|c|c|}
\hline$* * * * * * *$ & Prévu bon & $\begin{array}{l}\text { Prévu } \\
\text { mauvais }\end{array}$ & Total & $\%$ correct & $* * * * * * * *$ \\
\hline Bon & $\mathrm{TP}$ & $\mathrm{FN}$ & $\mathrm{TP}+\mathrm{FN}$ & $\frac{(100 * T P)}{T P+F N}$ & Sensibilité \\
\hline Mauvais & FP & $\mathrm{TN}$ & $\mathrm{FP}+\mathrm{TN}$ & $\frac{(100 * T N)}{F P+T N}$ & Spécificité \\
\hline Total & $\mathrm{TP}+\mathrm{FP}$ & $\mathrm{FN}+\mathrm{TN}$ & $* * * * * *$ & $* * * * * * *$ & $* * * * * *$ \\
\hline$\%$ correct & $\frac{(100 * T P)}{F P+T P}$ & $\frac{(100 * T N)}{T N+F N}$ & $* * * * * *$ & $\frac{(100 *(T P+T N))}{T P+F N+F P+T N}$ & $* * * * * *$ \\
\hline$* * * * * *$ & Précision & $* * * * * *$ & $* * * * * *$ & $\begin{array}{c}\text { \% total correctement } \\
\text { prévu }\end{array}$ & $* * * * * *$ \\
\hline
\end{tabular}

\subsection{L'analyse discriminante et l'arbre de décisions}

\subsubsection{Définitions}

L'analyse discriminante est le modèle le plus connu pour la prédiction de la défaillance grâce à sa forme la plus simple, elle vise à trouver une fonction linéaire pour les variables comptables et non comptables qui distinguent le mieux entre deux catégories d'emprunteurs, c'est-à-dire ceux qui vont honorer leurs créances et ceux qui ne vont pas rembourser leur emprunt. Elle se fond sur le principe de minimisation de la variance entre les groupes et la maximiser dans les groupes. Altman (1968) est la première personne à fournir des modèles de prédiction de la faillite multi-variables. Application multiple méthode d'analyse de détachement et en utilisant des ratios financiers comme variables indépendantes, il cherchait à prédire la faillite des instituts. Il a présenté son fameux motif intitulé Zscore, qui est bien connu dans la prédiction de la négociation faillite. Dans son étude, Altman a réuni 
un échantillon comprenant 66 entreprises productives pour la période 1946-1965 par la conception en utilisant la société non-faillite selon la faillite. Après l'analyse des données de cinq ratios de ratios financiers les plus importants ont été sélectionnés pour prédire modèle. Si Z-score est faible, la situation financière indésirable se produira et si le grade est élevé, il montre bon état. Le modèle a rapporté la précision de prédiction de $95 \%$ pour un an et $72 \%$ pour les deux années précédant la faillite. En comparant les résultats obtenus par l'étude Beaver, Altman a conclu que le modèle de prédiction basé sur une analyse multiple détachement a la capacité de fournir une meilleure prédiction pour différencier les entreprises en faillite et non en faillite par rapport à l'analyse à variables multiples. $^{5}$

Un arbre de décision est un instrument d'aide pour la prise de décision et à l'exploration de données. Il permet de représenter l'ensemble des cas possible sous forme d'un schéma.

Une technique de classification :

- créer des groupes homogènes au regard d'un certain nombre de variables actives.

- Permet de produire directement une règle d'affectation

- Permet de produire directement une règle d'affectation « industrialisable»

- Interprétation directe des groupes à l'aide des mêmes règles

- Rapidité/capacité à traiter de grandes bases (similaire aux arbres de décision)

- Possibilité de guider la recherche des classes

\section{Structure d'un arbre de décision}

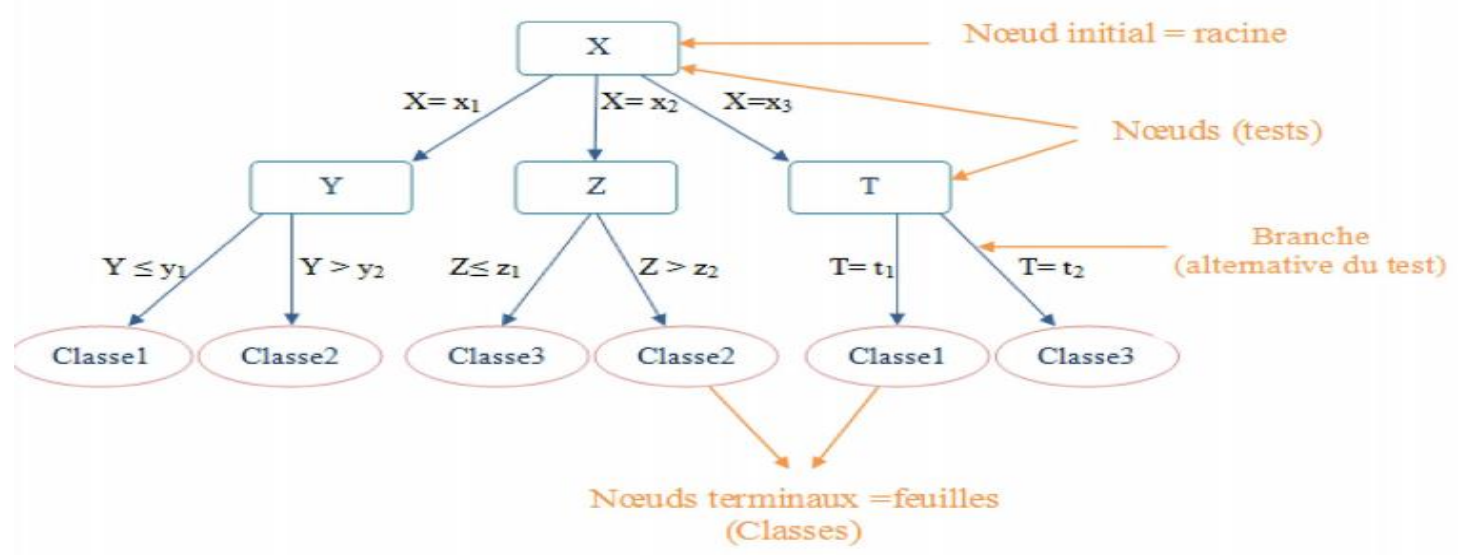

Figure 5 : Structure d'un arbre de décision.

\footnotetext{
${ }^{5}$ Ahmad AHMADPOUR KASGARI, Seyyed Hasan SALEHNEZHAD, Fatemeh EBADI. A Review of Bankruptcy and its Prediction Vol. 3, No. 4, October 2013, pp. 274-277, E-ISSN: 2225-8329, P-ISSN: 23080337, (C) 2013 HRMARS, www.hrmars.com.
} 


\subsubsection{La typologie des arbres}

\section{Les arbres CART :}

CART (Classification And Regression Trees) est un arbre binaire utilisé pour tout type des variables explicatives, il est un parmi les arbres les plus performants et les plus répandus, on le trouve notamment dans les logiciels : SAS, R, S Plus, SPAD et SPSS.

\section{Les arbres CHAID :}

CHAID (Chi-squared Automatic Interaction Detector), est un arbre parmi les premiers à avoir été implémenté dans des logiciels commerciaux. Il est efficace lorsque la taille de la base de données est importante et lorsque les variables explicatives sont qualitatives ou discrètes.

\section{Les arbres QUEST :}

QUEST est une technique qui est très utile avec les variables indépendantes avec de nombreuses modalités par rapport au biais des autres méthodes.

\subsubsection{Comparaison :}

Tableau 7 : Comparaison entre les arbres de décision

\begin{tabular}{|l|l|l|l|}
\hline & CHAHID & CRT & QUEST \\
\hline Calculé à partir du chi-deux & $\mathrm{X}$ & & \\
\hline $\begin{array}{l}\text { Variables (explicatives) } \\
\text { indépendantes de substitution }\end{array}$ & & $\mathrm{X}$ & $\mathrm{X}$ \\
\hline Elagage des arbres & & $\mathrm{X}$ & $\mathrm{X}$ \\
\hline Scission de nœud multiple & $\mathrm{X}$ & & \\
\hline Scission de nœud binaire & & $\mathrm{X}$ & $\mathrm{X}$ \\
\hline Variables d'influence & $\mathrm{X}$ & $\mathrm{X}$ & \\
\hline Probababilités à priori & & $\mathrm{X}$ & $\mathrm{X}$ \\
\hline Coûts de mauvaise réaffectation & $\mathrm{X}$ & $\mathrm{X}$ & $\mathrm{X}$ \\
\hline Calcul rapide & $\mathrm{X}$ & & $\mathrm{X}$ \\
\hline
\end{tabular}

Dans notre cas on va travailler sur l'arbre CHAHID car il remplis les conditions de notre analyse, et quelle est appliquer dans le cas où il y a un mélange entre des varibles qualitatives et quantitatives c'est le cas pour notre travail, on a la faillite un variable qualitative et les ratios sont des variables quantitatives. 


\section{Analyse empirique :}

Dans cette deuxième section nous présenterons les différentes données nécessaires pour notre étude, et les critères de choix des données les plus adéquates pour construire un modèle de prévision de la défaillance des entreprises. L'objectif espéré du modèle que nous allons construire est qu'il puisse nous permettre d'estimer est ce que l'entreprise est en faillite selon les données associées à chaque entreprise en comparant la capacité prédictive des trois techniques utilisé, la régression logistique, l'analyse discriminante et l'arbre de décision, pour savoir laquelle de ces trois méthodes est efficace pour la prévision de la défaillance. Pour pouvoir répondre à ce besoin, la sélection des variables qui vont contribuer à l'élaboration de ce modèle devrait traduire les éléments qui expliquent le mieux le phénomène étudié. On peut en général utiliser 2 catégories de variables:

- Variables comptables et financières, qui permettent de construire des Ratios retraçant la santé financière de l'entreprise.

- Variables de comportement managériaux, qui traduit la capacité de gestion de l'entreprise.

Compte tenu de la difficulté de l'obtention des variables de dernier catégorie, nous allons nous concentrer sur les variables financières nécessaires pour la constitution du modèle.

\subsection{Méthodologie}

Notre démarche de collecte des données comporte deux étapes : le choix des entreprises et des indicateurs de défaillance financière.

\section{La constitution de l'échantillon}

Pour établir notre échantillon nous avons utilisé une base de données obtenue du haut-commissariat au plan. On peut dire qu'une entreprise est en faillite dès lors qu'elle aura une première déclaration judicaire au tribunal de commerce entre la période de 2012 et 2014 et qui englobe la ville de Fès, rabat et Casablanca. L'échantillon final obtenu au terme de ce processus de sélection rigoureux se compose de 46 entreprises divisées en deux sous-échantillons : 25 entreprises saines et 21 entreprises défaillantes.

\section{Le choix des indicateurs ou des ratios financiers}

$\mathrm{Au}$ début on a demandé 10 ratios avec d'autres indicateurs financiers (comme $\mathrm{CA}$, nombre d'employés,...), nous n'avons pas reçues tous les variables demandées, Cette différence est due essentiellement à l'indisponibilité de quelques-unes des variables demandées, et aussi le problème de confidentialité des données. Nous avons finalement choisi d'utiliser que 4 ratios obtenus et qui sont couramment utilisés dans les travaux sur la prédiction de la faillite des entreprises.

Les ratios obtenus sont : 
Ratio de couverture de la dette totale par les flux de trésorerie

$\mathrm{R} 1=$ Flux de trésorerie / Dette totale

C'est un indicateur qui nous donne une idée sur la capacité de rembourser la dette totale

Le ROA (Return On Assets)

R2=Résultat net/ Actif

Il mesure l'efficacité de l'entreprise à générer des bénéfices

$>$ Le ratio fonds de roulement

R3= Actif à court terme/ Dette à court terme

C'est un indicateur qui permet à l'entreprise de savoir s'elle dispose d'un fonds de roulement suffisant pour couvrir ses obligations à court terme

Ratios de rentabilité

$\mathrm{R} 4=$ Actif à court terme/ Ventes

Pour évaluer si l'entreprise est rentable

La comparaison entre la capacité prédictive de l'analyse discriminante, la régression logistique, et les arbres de décisions a été réalisée sur la base des ratios financiers spécifiques à chaque entreprise.

Les ratios choisis sont observés sur 46 entreprises:

$\mathrm{X} 1=$ Flux de trésorerie $/$ Dette totale

X2=Résultat net/ Actif

X3= Actif à court terme/ Dette à court terme

$\mathrm{X} 4=$ Actif à court terme/ Ventes

La variable à exprimer est :

$\mathrm{Y}=\mathrm{F}$ si faillite, NF sinon

Faillite

\begin{tabular}{|c|c|c|c|c|c|}
\hline & & Effectifs & Pourcentage & $\begin{array}{c}\text { Pourcentage } \\
\text { valide }\end{array}$ & $\begin{array}{c}\text { Pourcentage } \\
\text { cumulé }\end{array}$ \\
\hline \multirow{3}{*}{ Valide } & $\mathrm{F}$ & 21 & 45,7 & 45,7 & 45,7 \\
\hline & $\mathrm{NF}$ & 25 & 54,3 & 54,3 & 100,0 \\
\hline & Total & 46 & 100,0 & 100,0 & \\
\hline
\end{tabular}

Tableau 8 : description de la base de données 
On a 46 observations présentant $54,3 \%$ des entreprises saines contre $45,7 \%$ des entreprises ont fait faillite donc une modélisation est nécessaire pour étudier cette défaillance.

\subsection{1. Évaluation de la capacité discriminante des variables}

La liste des ratios calculés constitue une base d'indicateurs intéressants pour évaluer les performances des entreprises de notre base. Cependant, les ratios qui doivent entrer dans la construction de notre modèle doivent respecter certaines conditions, notamment leur capacité à discriminer entre la population des entreprises en faillite et celle des saines, aussi ces indicateurs doivent-ils être dé corrélés afin d'éviter toute redondance d'informations. Alors, et afin de faire valoir ces conditions, nous avons effectué les traitements suivants :

\section{Utilisation du Courbe de roc}

Pour pouvoir juger la capacité discriminante de chaque variable, on a choisi d'utiliser la Courbe de roc dont le fonctionnement que nous avons expliqué précédemment, on a opté pour cette méthode grâce à son efficacité en termes de résultats.

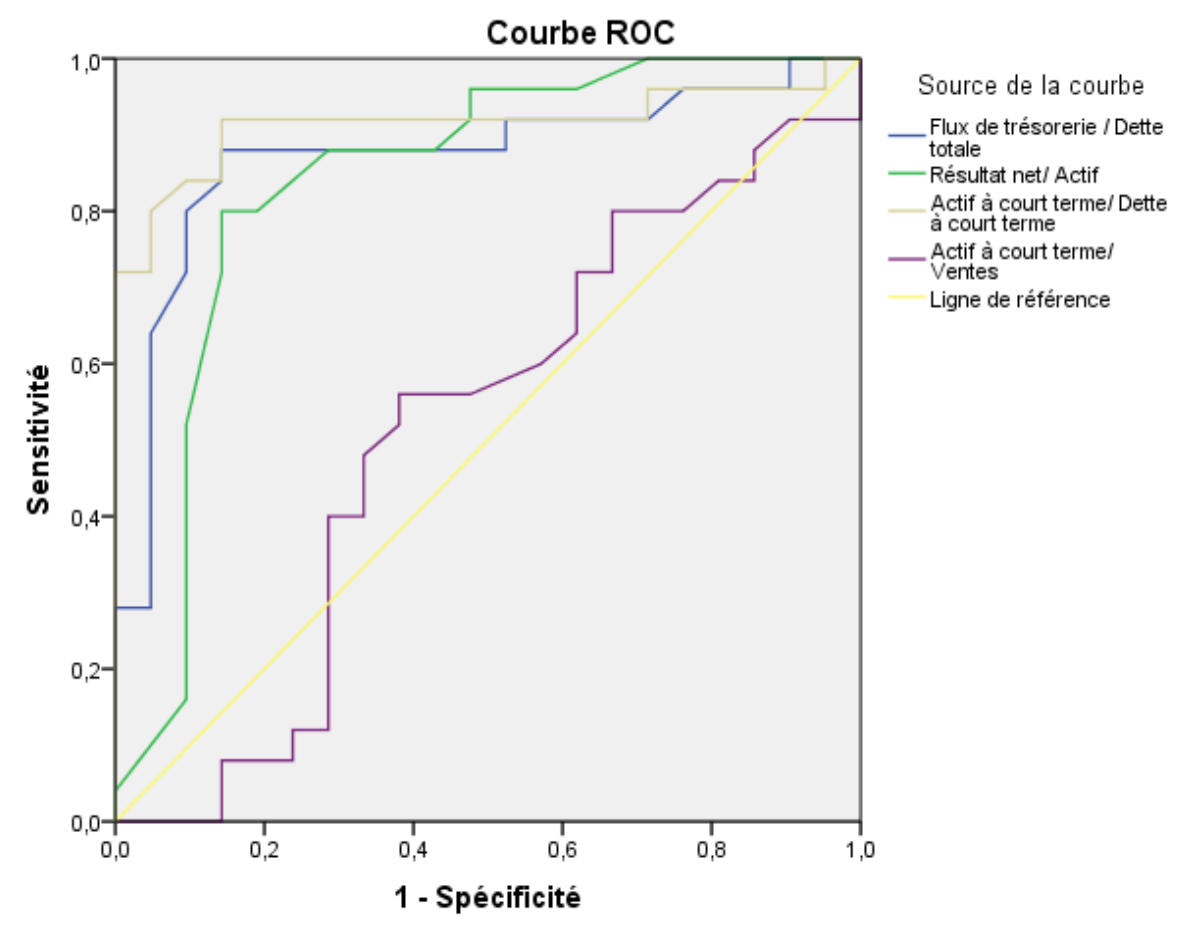

Les segments diagonaux sont générés par des liaisons.

Figure 6 : La courbe de roc des ratios retenus 


\section{Zone sous la courbe}

\begin{tabular}{|l|r|}
\hline Variable(s) de résultats tests & Zone \\
\hline Flux de trésorerie / Dette totale &, 872 \\
Résultat net/ Actif &, 842 \\
Actif à court terme/ Dette à court terme &, 915 \\
& \\
Actif à court terme/ Ventes &, 514 \\
& \\
\hline
\end{tabular}

Tableau 9 : zone sous la courbe

Puisque tous les ratios ont une zone supérieure à 0,5 , donc les variables explicatives sont bien en liaison avec la variable à expliquer (faillite). L'air sous la de courbe roc est une mesure du pouvoir prédictif de la variable X (R1, R2, R3, R4). Dans notre cas l'air de la zone sous la courbe est supérieur à 0,5 pour tous les variables $\mathrm{X}$, ce qui nous laisse avancer que nos variables $\mathrm{X}$ ont un pouvoir de prédiction fort.

Après le classement des variables par leur capacité discriminante, on a procédé à l'étude de corrélations des variables

\section{La corrélation}

Après l'étape de l'évaluation du pouvoir discriminant des variables à travers la Courbe de roc, nous avons procédé à l'étude de la corrélation entre les différents ratios afin d'éliminer la redondance de l'information. Nous avons examiné la matrice de la corrélation en considérant qu'une corrélation supérieure à 0.5 entre deux variables est intolérable.

Le critère d'élimination de l'une des deux correspond à celle qui a la valeur la plus faible de la courbe de roc. 


\begin{tabular}{|c|c|c|c|c|c|}
\hline \multicolumn{6}{|c|}{ Corrélations } \\
\hline & & $\begin{array}{c}\text { Flux de } \\
\text { trésorerie / } \\
\text { Dette totale }\end{array}$ & $\begin{array}{l}\text { Résultat net/ } \\
\text { Actif }\end{array}$ & $\begin{array}{l}\text { Actif à court } \\
\text { terme/ Dette à } \\
\text { court terme }\end{array}$ & $\begin{array}{l}\text { Actif à court } \\
\text { terme/ Ventes }\end{array}$ \\
\hline \multirow{4}{*}{$\begin{array}{l}\text { Flux de trésorerie / Dette } \\
\text { totale }\end{array}$} & Corrélation de Pearson & 1 &, $823^{* *}$ &, $547^{* *}$ &,- 079 \\
\hline & Sig. (bilatérale) & &, 000 &, 000 & ,600 \\
\hline & $\mathrm{N}$ & 46 & 46 & 46 & 46 \\
\hline & Corrélation de Pearson &, $823^{* *}$ & 1 &, $471^{* *}$ &, 055 \\
\hline \multirow[t]{2}{*}{ Résultat net/ Actif } & Sig. (bilatérale) &, 000 & &, 001 &, 717 \\
\hline & $\mathrm{N}$ & 46 & 46 & 46 & 46 \\
\hline \multirow{3}{*}{$\begin{array}{l}\text { Actif à court terme/ Dette à } \\
\text { court terme }\end{array}$} & Corrélation de Pearson &, $547^{* *}$ &, $471^{* *}$ & 1 &, 154 \\
\hline & Sig. (bilatérale) &, 000 &, 001 & & ,306 \\
\hline & $\mathrm{N}$ & 46 & 46 & 46 & 46 \\
\hline \multirow{3}{*}{ Actif à court terme/ Ventes } & Corrélation de Pearson &,- 079 &, 055 &, 154 & 1 \\
\hline & Sig. (bilatérale) & ,600 & ,717 & ,306 & \\
\hline & $\mathrm{N}$ & 46 & 46 & 46 & 46 \\
\hline
\end{tabular}

** La corrélation est significative au niveau 0.01 (bilatéral).

Tableau 10 : Corrélation

D'après la matrice de corrélation on trouve que $\mathrm{X} 1$ et très corrélé avec la variable $\mathrm{X} 2$ avec une valeur de 0,82 . Le critère d'élimination de l'une des deux correspond à celle qui a la valeur la plus faible de la courbe de roc.

D'après le tableau de roc on va éliminer la variable X2 et garder la variable X1

Finalement on va continuer notre étude par les variables X1, X3 et X4.

\subsubsection{Résultats de la régression logistique}

Pour tester le pouvoir prédictive de la régression logistique, la première étape est de tester

l'importance des variables utilisés on utilisant le test de Wald, pour mesurer la qualité du modèle on utilise le coefficient de corrélation, et pour tester si le modèle est validé on utilise le taux de bon classement et le test de Kappa.

\section{Tests de plus-value (le test de Wald)}

Le test de Wald est utilisé pour tester l'importance de chaque ratio, dans cette étape on va tester si la constante est capable pour expliquer le modèle. 


\begin{tabular}{|c|c|c|c|c|c|c|c|}
\hline \multicolumn{8}{|c|}{ Variables dans l'équation } \\
\hline & & A & E.S. & Wald & Ddl & Sig. & $\operatorname{Exp}(B)$ \\
\hline étape 0 & Constante & ,174 & 296 &, 347 & 1 & ,556 & 1,190 \\
\hline
\end{tabular}

Tableau 11: du test global du Wald

D'après ce test on a trouvé la signification est supérieur à 0.05 , elle est égale à 0.556 . Donc la constante seule est incapable d'expliquer le modèle.

\section{Construction du modèle}

Après avoir testé le modèle et les variables retenues, on va présenter notre modèle.

Les coefficients de notre modèle sont élaborés par un programme Spss et présentés comme suit :

Variables dans l'équation

\begin{tabular}{|rl|r|r|r|r|r|r|}
\hline & \multicolumn{1}{|c|}{ A } & \multicolumn{1}{c|}{ E.S. } & \multicolumn{1}{|c|}{ Wald } & Ddl & \multicolumn{1}{c|}{ Sig. } & \multicolumn{1}{|c|}{$\operatorname{Exp(B)}$} \\
\hline & X1 & 5,753 & 3,050 & 3,559 & 1 &, 059 & 315,283 \\
& X3 & 3,332 & 1,086 & 9,413 & 1 &, 002 & 28,004 \\
étape $1^{\mathrm{a}}$ & & $-3,013$ & 3,050 &, 976 & 1 &, 323 &, 049 \\
& X4 & $-5,121$ & 2,059 & 6,185 & 1 &, 013 &, 006 \\
\hline
\end{tabular}

a. Variable(s) entrées à l'étape $1: \mathrm{X} 1, \mathrm{X} 3, \mathrm{X} 4$.

Tableau 13: Estimation des coefficients du modèle

Donc, notre modèle s'écrit de façon suivante :

$$
P(Y=F / X)=\frac{\exp \left(-5.121+5,753 X_{1}+3,332 X_{3}-3.013 X_{4}\right)}{1-\exp \left(-5.121+5,753 X_{1}+3,332 X_{3}-3.013 X_{4}\right)}
$$

\section{Mesure de qualité du modèle}

Après avoir testé l'interprétation des coefficients, on a mesuré la qualité de modélisation par les pseudos $\mathrm{R}$ mentionnés précédemment, on a eu les deux pseudos $R 2$ qui nous permettent d'expliquer le pourcentage de la variable dépendante binaire, Le Nagelkerke est une version ajustée du Cox Snell et est donc plus près de la réalité. 
Récapitulatif des modèles

\begin{tabular}{|r|r|r|r|}
\hline Étape & $\begin{array}{c}-2 \log - \\
\text { vraisemblance }\end{array}$ & $\begin{array}{c}\text { R-deux de Cox } \\
\& \text { Snell }\end{array}$ & R-deux de Nagelkerke \\
\hline 1 & $27,690^{\mathrm{a}}$ &, 540 &, 722 \\
\hline
\end{tabular}

Tableau 14: Test de $\mathrm{R}^{2}$

Ainsi, 72,2\% de la variation dans la faillite pourrait être expliquée.

\section{Validation du modèle}

Pour tester le modèle si validé ou pas, on utilise le taux de bon classement et l'indice de Kappa :

\section{Taux de bon classement du modèle}

Pour mesurer un modèle, parmi les performances qu'on doit examiner, c'est le taux de bon classement lié à la règle de décision encore appelée règle d'affectation. Plus le taux de bon classement est élevé plus le modèle est bon.

Le taux de bon classement dans l'échantillon d'apprentissage

Tableau de classement

\begin{tabular}{|c|c|c|c|c|}
\hline & \multirow[t]{3}{*}{ Observations } & \multicolumn{3}{|c|}{ Prévisions } \\
\hline & & \multicolumn{2}{|c|}{ Faillite } & \multirow{2}{*}{$\begin{array}{c}\text { Pourcentage } \\
\text { correct }\end{array}$} \\
\hline & & $\mathrm{F}$ & NF & \\
\hline \multirow{3}{*}{ Étape 1} & $\mathrm{~F}$ & 19 & 2 & 90,5 \\
\hline & NF & 1 & 24 & 96,0 \\
\hline & Pourcentage global & & & 93,5 \\
\hline
\end{tabular}

a. La valeur de césure est ,500

Tableau 15 : bon classement de la régression logistique

- Le taux de bon classement des entreprises saines : $96 \%$.

- Le taux de bon classement des entreprises en défaut : 90,5\%.

Selon ce tableau, le modèle a réalisé un taux de bon classement global de 93,5\%, c'est-à-dire que sur 100 entreprises 93 seront classées correctement et 7 seront mal classées par ce modèle, ce qui est très satisfaisant comme résultat. 


\section{Test du kappa}

A partir du tableau de classement on va calculer l'indice de kappa

$p($ correct $)=\frac{(T P+T N)}{(T P+T N+F P+F N)}=\frac{19+24}{19+2+1+24}=0,934$

$p($ hasard $)=\frac{\left(T P-\frac{(T P+F P)}{2}\right)+\left(T N-\left(\frac{(T N+F N)}{2}\right.\right.}{(T P+T N+F P+F N)}=\frac{\left(19-\left(\frac{19+1}{2}\right)+\left(24-\left(\frac{24+2}{2}\right)\right.\right.}{19+2+1+24}=0,434$

Kappa $=100 \times \frac{p(\text { correct })-p(\text { hasard })}{1-p(\text { hasard })}=100 \times \frac{0,934-0,434}{1-0,434}=88,3 \%$

Donc, le classement est bon, Ce qui est montre que notre modèle à une capacité de discrimination forte.

\section{Courbe de ROC}

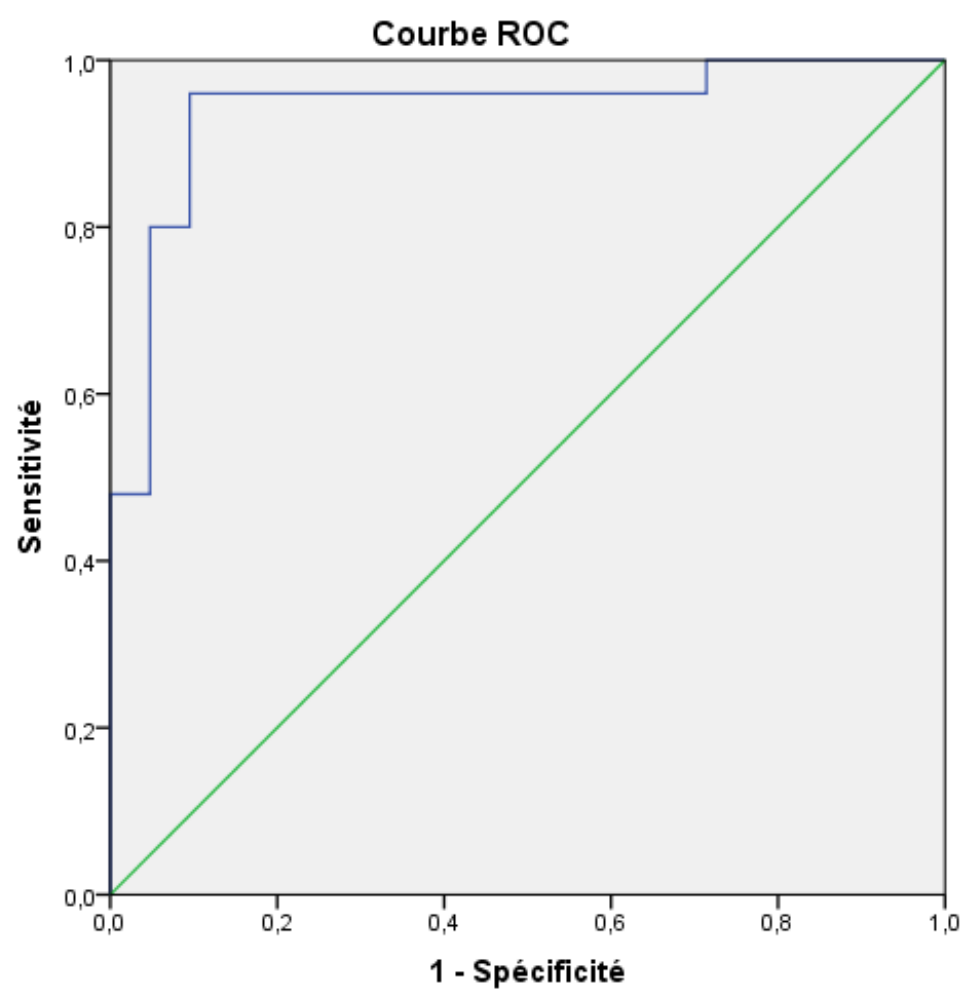

Figure 7 : courbe de ROC 
L'air sous la courbe de ROC est une mesure de pouvoir prédictif de la variable X, dans notre cas l'air de la zone sous courbe est égale à $94,1 \%$, ce qui nous laisse avancer que notre modèle à une capacité de discrimination fort.

\subsection{Résultats}

\subsubsection{Résultat de la régression logistique}

D'après le test de Wald, les ratios utilisé sont très significatives et qui nous a donnée des résultats pertinente en terme de prévision de la régression logistique, pour mesurer la qualité du modèle on a utilisé le coefficient de corrélation, sa valeur est égale à 0.72 qui proche de 1 ce qui signifie que notre modèle est pertinent, le test de taux de bon classement est valable pour la validation du modèle, on a trouvé pour le taux de bon classement que le modèle a réalisé est de 93,5\% et de même pour le test de kappa qui est égale à $79.3 \%$, ce classement est bon. Chose qui nous confirme que notre modèle de régression logistique est assez consistant en capacité de discrimination, ce qui montre que la régression logistique à une capacité prédictive très forte.

\subsubsection{Résultat de la méthode des arbres de décision}

On a utilisé les arbres CHAID qui répond bien à notre étude

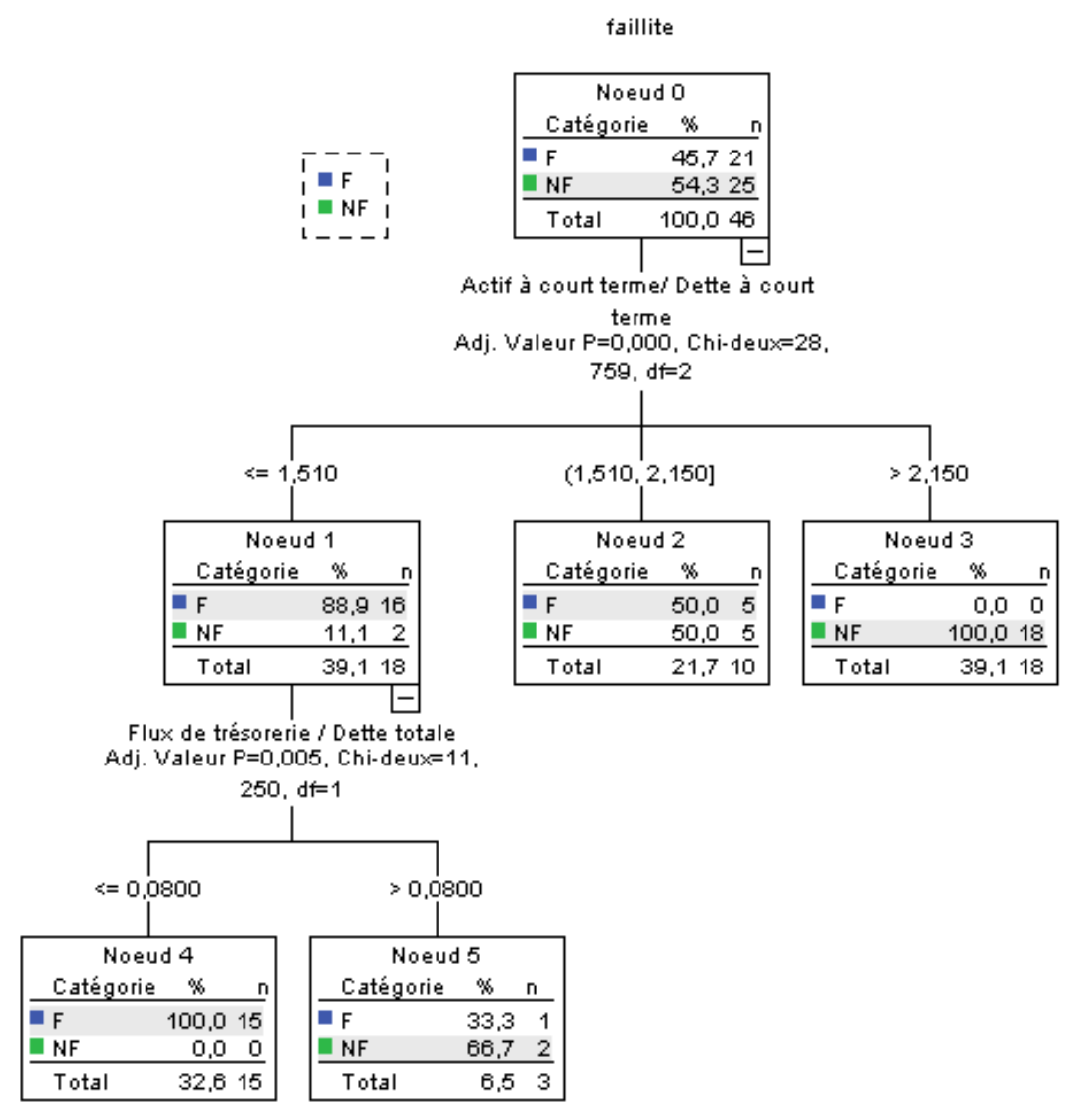

Figure 8 : Arbre de décision CHAID. 
D'après l'arbre de décision on trouve que la variable de segmentation la plus importante c'est la variable X3=Actif à court terme/ Dette à court terme.

Tableau descriptive :

\begin{tabular}{|l|l|l|}
\hline \multirow{2}{*}{ Noud } & Résultat & Non faillite \\
\cline { 2 - 3 } & Faillite & $0 \%$ \\
\hline$(\mathrm{X} 3 \leq 1,51, \mathrm{X} 1 \leq 0,8)$ & $100 \%$ & $66,7 \%$ \\
\hline$(\mathrm{X} 3 \leq 1,51, \mathrm{X} 1>0,8)$ & $33,3 \%$ & $50 \%$ \\
\hline $\mathrm{X} 3 \in] 1,51,2,15]$ & $50 \%$ & $100 \%$ \\
\hline $\mathrm{X} 3>2,15$ & & $0 \%$ \\
\hline
\end{tabular}

Tableau 16 : description de l'arbre de décision

D'après le tableau on trouve que si la variable X $3 \leq 1,51$ et la variable $\mathrm{X} 1 \leq 0,8$ l'entreprise est $100 \%$ en faillite. Et si la variable X3>2,15 l'entreprise est 100\% saine.

\section{Risque}

\begin{tabular}{|c|c|}
\hline Estimation & Erreur standard \\
\hline, 130 &, 050 \\
\hline
\end{tabular}

Méthode de développement : CHAID

Variable dépendante : faillite

Le risque d'erreur de l'arbre de décision c'est $5 \%$ ce qui est bien comme résultat.

\section{Validation de modèle :}

Pour valider le modèle de l'arbre de décision, on utilise le taux de bon classement et le test de kappa.

Taux de bon classement :

\begin{tabular}{|c|c|c|c|}
\hline \multicolumn{4}{|c|}{ Classification } \\
\hline \multirow[t]{2}{*}{ Observations } & \multicolumn{3}{|c|}{ Prévisions } \\
\hline & $\mathrm{F}$ & NF & Pourcentage correct \\
\hline $\mathrm{F}$ & 20 & 1 & $95,2 \%$ \\
\hline NF & 5 & 20 & $80,0 \%$ \\
\hline Pourcentage global & $54,3 \%$ & $45,7 \%$ & $87,0 \%$ \\
\hline
\end{tabular}

Méthode de développement : CHAID

Variable dépendante : faillite

Tableau 17: bon classement de l'arbre de décision 
- Le taux de bon classement des entreprises saines : $80 \%$

Le taux de bon classement des entreprises en défaut : 95,2\%. Selon ce tableau, le modèle a réalisé un taux de bon classement global de 87\%, c'est-à-dire que sur 100 entreprises 87 seront classées correctement et 13 seront mal classées par ce modèle, ce qui est très satisfaisant comme résultat.

\section{L'indice de Kappa :}

On a $p($ correct $)=\frac{(T P+T N)}{(T P+T N+F P+F N)}=\frac{20+20}{20+1+5+20}=0,87$

$p($ hasard $)=\frac{\left(T P-\frac{(T P+F P)}{2}\right)+\left(T N-\left(\frac{(T N+F N)}{2}\right.\right.}{(T P+T N+F P+F N)}=\frac{\left(20-\left(\frac{20+5}{2}\right)+\left(20-\left(\frac{20+1}{2}\right)\right.\right.}{20+1+5+20}=0,37$

Kappa $=100 \times \frac{p(\text { correct })-p(\text { hasard })}{1-p(\text { hasard })}=100 \times \frac{0,87-0,37}{1-0,37}=79,3 \%$

Donc, le classement est bon. Chose qui nous confirme que notre modèle est assez consistant en capacité de discrimination. Pour l'arbre de décision Le taux de bon classement des entreprises en défaut : 95,2\%, le modèle a réalisé un taux de bon classement global de $87 \%$, c'est-àdire que sur 100 entreprises 87 seront classées correctement et 13 seront mal classées par ce modèle, ce qui est très satisfaisant comme résultat.

\subsubsection{Résultat de la méthode d'analyse discriminante}

Première étape consiste à chercher s'il existe des différences entre les groupes à l'aide de trois indicateurs: la moyenne, le test du F et le Lambda de Wilks. Qui nous permet de sélectionner les variables les plus discriminantes. Leur interprétation se fait de la façon suivante:

\begin{tabular}{|l|l|l|}
\hline & En cas d'influence & En absence d'influence \\
\hline Moyenne ou variable & Différence & Similitude \\
\hline Test du F & F élevé & F faible \\
& Signification F tend vers 0,000 & Signification F $>=0,01$ ou 0,05 \\
& & Tend vers 1 \\
\hline Lambda de Wilks & $<=0,90$ & \\
\hline
\end{tabular}

Tableau 18 : mesure de l'influence entre les variables.

Les moyennes s'observent dans le tableau «Statistiques de groupe». Les variables $\mathrm{X} 1, \mathrm{X} 3$ dans le tableau ci-dessous sont les variables les plus discriminantes. 
Statistiques de groupe

\begin{tabular}{|c|c|c|c|c|c|}
\hline \multicolumn{2}{|c|}{ Faillite } & \multirow[t]{2}{*}{ Moyenne } & \multirow[t]{2}{*}{ écart-type } & \multicolumn{2}{|c|}{$\mathrm{N}$ valide (liste) } \\
\hline & & & & Non pondérées & Pondérées \\
\hline \multirow{3}{*}{$\mathrm{F}$} & Flux de trésorerie / Dette totale &,- 05300 & ,206378 & 21 & 21,000 \\
\hline & Actif à court terme/ Dette à court terme & 1,36667 & ,405343 & 21 & 21,000 \\
\hline & Actif à court terme/ Ventes & ,43762 & ,211138 & 21 & 21,000 \\
\hline \multirow{3}{*}{$\mathrm{NF}$} & Flux de trésorerie / Dette totale &, 23520 & ,216912 & 25 & 25,000 \\
\hline & Actif à court terme/ Dette à court terme & 2,59360 & 1,023120 & 25 & 25,000 \\
\hline & Actif à court terme/ Ventes &, 42680 &, 162422 & 25 & 25,000 \\
\hline \multirow{3}{*}{ Total } & Flux de trésorerie / Dette totale & ,10363 &, 255126 & 46 & 46,000 \\
\hline & Actif à court terme/ Dette à court terme & 2,03348 & 1,006528 & 46 & 46,000 \\
\hline & Actif à court terme/ Ventes & ,43174 &, 184153 & 46 & 46,000 \\
\hline
\end{tabular}

Tableau 19: Statistiques de groupe

Le test du F et du Lambda de Wilks s'observe dans le tableau «Tests d'égalité des moyennes des groupes». On trouve que la signification de test $F$ tend vers 0 lambda de wilks $<=0,90$ pour les deux variables X1 et X3 cela nous confirme que ce sont bien les variables X1 et X3 qui sont les plus discriminantes.

Tests d'égalité des moyennes des groupes

\begin{tabular}{|l|r|r|r|r|r|}
\hline & \multicolumn{1}{|c|}{$\begin{array}{c}\text { Lambda de } \\
\text { Wilks }\end{array}$} & F & ddl1 & ddl2 & Signification \\
\hline $\begin{array}{l}\text { Flux de trésorerie / Dette } \\
\text { totale }\end{array}$ &, 676 & 21,054 & 1 & 44 &, 000 \\
$\begin{array}{l}\text { Actif à court terme/ Dette à } \\
\text { court terme }\end{array}$ &, 623 & 26,610 & 1 & 44 &, 000 \\
Actif à court terme/ Ventes &, 999 &, 039 & 1 & 44 &, 845 \\
\hline
\end{tabular}

Tableau 20 : Tests d'égalité des moyennes des groupes

\section{Vérification de la validité de l'étude}

On estime la validité de l'analyse discriminante par trois indicateurs :

- Le test de Box.

- La corrélation globale.

- Le Lambda de Wilks.

Le test de Box grâce au tableau «Résultats du test» 
Résultats du test

\begin{tabular}{|l|r|r|}
\hline M de Box & & 20,708 \\
& Approximativement & 3,192 \\
F & ddl1 & 6 \\
& ddl2 & 12870,997 \\
& Signification &, 004 \\
\hline
\end{tabular}

Teste l'hypothèse nulle d'égalité de matrices de covariance des populations.

Tableau 21: Résultats du test de box.

Le $\mathrm{M}$ doit être plus élevé. La significativité du test de $\mathrm{F}$ doit tendre vers 0 et inférieur à 0,05 pour confirmer la validité de l'analyse.

Puisque le sig $=0,004<0,05$ donc l'analyse de discriminante est validée

La corrélation globale se mesure quant à elle, se retrouve dans le tableau « Eigenvalues » (Valeurs propres)

Valeurs propres

\begin{tabular}{|r|r|r|r|r|}
\hline Fonction & Valeur propre & $\begin{array}{c}\text { \% de la } \\
\text { variance }\end{array}$ & \% cumulé & Corrélation canonique \\
\hline 1 &, $851^{\mathrm{a}}$ & 100,0 & 100,0 &, 678 \\
\hline
\end{tabular}

a. Les 1 premières fonctions discriminantes canoniques ont été utilisées pour l'analyse.

Tableau 22: Valeurs propres

On observe la colonne «Corrélation canonique» plus elle est proche de 1 , on dit que notre modèle est meilleur.

Dans notre cas la corrélation égale à 0,68 ce qui est satisfaisante comme résultat

Lambda de Wilks

\begin{tabular}{|l|r|r|r|r|}
\hline $\begin{array}{l}\text { Test de la ou des } \\
\text { fonctions }\end{array}$ & $\begin{array}{c}\text { Lambda de } \\
\text { Wilks }\end{array}$ & Khi-deux & ddl & Signification \\
\hline 1 &, 540 & 26,165 & 3 &, 000 \\
\hline
\end{tabular}


Plus la valeur du Lambda de Wilks (deuxième colonne) est faible, et sa significativité tend vers 0 , plus le modèle est bon.

Dans notre cas on trouve que notre modèle est bon puisque la signification tend vers 0

\section{Estimation des coefficients de la fonction discriminante}

On observe la capacité discriminant des axes grâce au tableau «Coefficients des fonctions discriminantes canoniques».

\begin{tabular}{|l|c|}
\hline \multicolumn{2}{|c|}{ Coefficients des fonctions discriminantes canoniques } \\
\cline { 2 - 3 } & \multicolumn{2}{|c|}{ Fonction } \\
\hline $\begin{array}{l}\text { Flux de trésorerie / Dette totale } \\
\text { Actif à court terme/ Dette à court } \\
\text { terme }\end{array}$ & 1 \\
Actif à court terme/ Ventes & 2,453 \\
& \\
(Constante) & \\
\end{tabular}

Coefficients non standardisés

Tableau 23: Coefficients des fonctions discriminantes canoniques

Ce tableau permet d'obtenir la fonction discriminante. Dans notre exemple, la fonction discriminante est égale à :

$-1,724+2,453 \times X 1+0,893 \times X 3-0,802 \times X 4$

Tableau de bon classement :

\begin{tabular}{|c|c|c|c|c|c|}
\hline \multicolumn{6}{|c|}{ Résultats du classement ${ }^{\mathrm{a}, \mathrm{c}}$} \\
\hline & & \multirow[t]{2}{*}{ Faillite } & \multicolumn{2}{|c|}{ Classe(s) d'affectation prévue(s) } & \multirow[t]{2}{*}{ Total } \\
\hline & & & $\mathrm{F}$ & NF & \\
\hline \multirow{4}{*}{ Original } & & $\mathrm{F}$ & 19 & 2 & 21 \\
\hline & LIICClI & NF & 6 & 19 & 25 \\
\hline & & $\mathrm{F}$ & 90,5 & 9,5 & 100,0 \\
\hline & 0 & $\mathrm{NF}$ & 24,0 & 76,0 & 100,0 \\
\hline \multirow{4}{*}{ Validé-croiséb } & & $\mathrm{F}$ & 19 & 2 & 21 \\
\hline & 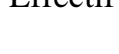 & $\mathrm{NF}$ & 6 & 19 & 25 \\
\hline & $o r$ & $\mathrm{~F}$ & 90,5 & 9,5 & 100,0 \\
\hline & & $\mathrm{NF}$ & 24,0 & 76,0 & 100,0 \\
\hline
\end{tabular}

a. $82,6 \%$ des observations originales classées correctement.

Tableau 24 : Bon classement de l'analyse discriminante. 
La note (a) nous indique le pouvoir de reclassement de la fonction discriminante, ici c'est $82,6 \%$ ce qui bien comme résultat.

Cette valeur se calcule comme suit:

$=($ taux bon classement de F+taux bon classement NF $) /$ Somme des entreprises

$=100 *(19+19) / 46=82,6 \%$

Concernant l'analyse discriminante, La première chose qu'on vérifier est ce que l'analyse discriminante a un influence ou pas sur les ratios, est ce que la faillite influence les ratios ou pas, on cherche la variable qui influence bien, pour cela on a trouvé pour lambda de wilks une valeur inférieur à 0.90 pour les flux de trésorerie/dette total et actif à court terme/dette à court terme donc ces deux ratios influencent bien et sont les plus discriminant. Pour test de box on a trouvé une valeur grande et sa signification est inférieur à 0.05 ce qui permet de conclure que l'analyse discriminante est valable pour notre cas et importante il nous a donné des résultats efficaces. Donc Pour l'analyse discriminante, le pouvoir de classement est de $82,6 \%$ ce qui bien comme résultat

\section{Comparaison :}

Dans l'ensemble des trois techniques de prédiction choisis : Analyse discriminante linéaire, la régression logistique, arbres de décisions offrent un pouvoir prédictif supérieur ou égal à $87 \%$. En se référant au tableau suivant, il est possible de dégager quelques éléments qui aident à choisir le meilleur modèle de prédiction de faillite des entreprises marocaines.

\begin{tabular}{|l|l|l|}
\hline Méthode & \multicolumn{1}{|c|}{ État } & \multicolumn{1}{|c|}{ Taux } \\
\hline \multirow{4}{*}{ Régression logistique } & Saines & $96 \%$ \\
\cline { 2 - 3 } & Faillite & $90,5 \%$ \\
\cline { 2 - 3 } & Bon classement & $93,5 \%$ \\
\hline \multirow{4}{*}{ Arbres de décisions } & Saines & $80 \%$ \\
\cline { 2 - 3 } & Faillite & $95,2 \%$ \\
\cline { 2 - 3 } Analyse discriminante & Bon classement & $87 \%$ \\
\hline & Saines & $76 \%$ \\
\cline { 2 - 3 } & Faillite & 90,5 \\
\cline { 2 - 3 } & Bon classement & $82,6 \%$ \\
\hline
\end{tabular}

Tableau 25: Tableau de comparaison entre les trois modèles de prévision de la faillite.

Le résultat obtenu dans le tableau ci-dessus nous permet de conclure que la méthode de la régression logistique est plus performante en comparaison avec les autres méthodes de l'analyse discriminante et l'arbre de décision. 


\section{Conclusion}

La faillite des entreprises demeure la principale préoccupation pour les entreprises et d'autres institutions financières qui ont mis beaucoup d'efforts en essayant de trouver les moyens les plus pertinents de contrôler ou atténuer ce risque.

Dans ce contexte, l'objectif principal de ce travail était de faire une comparaison entre les trois modèles de prévision de la faillite, la régression logistique, l'arbre de décision et l'analyse discriminante pour savoir la capacité prédictive de ces trois méthodes et qui est la meilleur méthode en comparaison avec les autres.

À ce sujet nous avons essayé d'avoir des connaissances sur la thématique de la défaillance, en testant trois modèles d'analyse statistique la régression logistique, l'analyse discriminante et l'arbre de décision, et on a trouvé que Les résultats obtenus sont relativement satisfaisants, puisque le taux de classification varie entre $87 \%$ et $93,5 \%$, pour l'analyse discriminante $82,6 \%$ pour la régression logistique $93,5 \%$, l'arbre de décisions $87 \%$. Ces résultats sont proches et même meilleurs que ceux qui découlent des études antérieures réalisées sur ce sujet. La méthode de la régression logistique a des résultats meilleurs en comparaison avec les autres méthodes de l'arbre de décision et l'analyse discriminante.

D'après le test de Wald, les ratios utilisé sont très significatives et qui nous a donnée des résultats pertinente en terme de prévision de la régression logistique, pour mesurer la qualité du modèle on a utilisé le coefficient de corrélation, sa valeur est égale à 0.72 qui proche de 1 ce qui signifie que notre modèle est pertinent, le test de taux de bon classement est valable pour la validation du modèle, on a trouvé pour le taux de bon classement que le modèle a réalisé est de $93,5 \%$ et de même pour le test de kappa qui est égale à $79.3 \%$, ce classement est bon. Chose qui nous confirme que notre modèle de régression logistique est assez consistant en capacité de discrimination, ce qui montre que la régression logistique à une capacité prédictive très forte. Pour l'arbre de décision Le taux de bon classement des entreprises en défaut : 95,2\%, le modèle a réalisé un taux de bon classement global de 87\%, c'est-à-dire que sur 100 entreprises 87 seront classées correctement et 13 seront mal classées par ce modèle, ce qui est très satisfaisant comme résultat.

Concernant l'analyse discriminante, La première chose qu'on vérifier est ce que l'analyse discriminante a un influence ou pas sur les ratios, est ce que la faillite influence les ratios ou pas, on cherche la variable qui influence bien, pour cela on a trouvé pour lambda de wilks une valeur inférieur à 0.90 pour les flux de trésorerie/dette total et actif à court terme/dette à court terme donc ces deux ratios influencent bien et sont les plus discriminant. Pour test de box on a trouvé une valeur grande et sa signification est inférieur à 0.05 ce qui permet de conclure que l'analyse discriminante est valable pour notre cas et importante il nous a donné des résultats efficaces. Donc Pour l'analyse discriminante, le pouvoir de classement est de $82,6 \%$ ce qui bien comme résultat. 
Ce résultat rejoint les résultats de certains travaux empiriques comparables Yang, Platt et Platt (1999), Lennox (1999).

Les résultats obtenus sont relativement satisfaisants, puisque le taux de classification varie entre $87 \%$ et $93,5 \%$, pour l'analyse discriminante $82,6 \%$ pour la régression logistique $93,5 \%$, l'arbre de décisions $87 \%$. Ces résultats sont proches et même meilleurs que ceux qui découlent des études antérieures réalisées sur ce sujet. La méthode de la régression logistique a des résultats meilleurs en comparaison avec les autres méthodes de l'arbre de décision et l'analyse discriminante.

\section{Références bibliographiques :}

\section{OUVRAGES ET REVUS}

- AHMADPOUR KASGARI Ahmad, Seyyed Hassan SALEHNEZHAD, Fatemeh EBADI. A Review of Bankruptcy and its Prediction .Vol. 3, No. 4, October 2013, pp. 274-277, E-ISSN: 2225-8329, P-ISSN: 2308-0337, 2013 HRMARS, <www.hrmars.com>.

- ALIAKBARI SAEIDEH, Prediction of Corporate Bankruptcy for the UK Firms in Manufacturing Industry, Department of Economics and Finance, School of Social Sciences MSc in Finance and Investment, Brunel University, September 2009.

- BLAZY et Al (1993) cité in : BLAZY Régis, CHARLETY Patricia, COMBIER Jérôme. Les défaillances d'entreprises : des difficultés visibles plusieurs années à l'avance. In: Economie et statistique, $\mathrm{N}^{\circ} 268-269,1993$. pp. 101-111.

- BLAZY Régis, CHARLETY Patricia, COMBIER Jérôme. Les défaillances d'entreprises : des difficultés visibles plusieurs années à l'avance. In: Economie et statistique, N²68-269, 1993. pp. 101-111.

- CHAHBI Ahmed, La détection des entreprises en difficulté, mémoire présenté en vue de l'obtention de diplôme nationale de l'expert-comptable.

- Commercial laws of Morocco, May 2013, an assessment by the ebrd, office of the general counsel, European bank.

- CRUTZEN et CAILLIE (2008):Le processus de défaillance de l'entreprise : Intégration et relecture de la littérature $\mathrm{P} 4$.

- FIMAYER Agnès, la détresse financière des entreprises, Trajectoire de déclin et traitement judiciaire de défaut.

- GADHOUM YOSER et al. « La décision de crédit. Procédure et comparaison de la performance de quatre modèles de prévision d'insolvabilité », La Revue des Sciences de Gestion 2007/2(n`224-225), p. 177-183.

- MANIANI (2006) : MAGHRESS, Revue électronique marocaine. 
- MARCO et RAINELLI (1986) : Les disparitions de firmes industrielles, Revue d'économie industrielle, PP.1-13.

- M'RABET et TAZI M (1991) : Les causes de la défaillance des entreprises

- ZOPOUNIDIS (1995), cité in La défaillance des entreprises: une revue de littérature, La défaillance des entreprises: une revue de littérature, <http://www.ipag.fr/fr/accueil/larecherche/publications-WP.html>.

\section{Webographie :}

- <http://www.meilleurtaux.ma/actualites/2015-novembre/hausse-des-previsions-dedefaillances-d-entreprises-au-maroc.html>.

<http://www.le360.ma/fr/economie/plus-de-8000-entreprises-menacees-de-faillite-29595>,Par Ismail BENBABA le 13/01/2015 à 08h03 (mise à jour le 13/01/2015 à 10h19).

- $\quad<$ Http://www.maroc-hebdo.press.ma/le-maroc-confronte-a-une-cascade-de-faillitesdentreprises/>.

- <http://www.challenge.ma/faillites-dentreprises-les-secteurs-a-surveiller-en-2015-37669>.

- <http://www.leconomiste.com/article/965084-analysepourquoi-les-pme-font-faillite>.

- <http://www.ipag.fr/fr/accueil/la-recherche/publications-WP.html>.

- <www.Inforisk.ma>. 